\title{
Interdisciplinary Analysis of Annual Space Weather Activity in Relation to Mortality Associated with Cerebrovascular Disease: A Novel Model of Solar-Terrestrial Interaction
}

\author{
Joseph M. Caswell ${ }^{1,2,3, a}$, Trevor N. Carniello ${ }^{1,2,4, b}$, \\ William F. T. Mekers $2,4, c$ \\ Transnational Anomalies Research ${ }^{1}$, Sudbury, Ontario, Canada, P3E3W6 \\ Neuroscience Research Group ${ }^{2}$, Human Studies ${ }^{3}$ and Biology ${ }^{4}$ Programs, Laurentian University, \\ Sudbury, Ontario, Canada, P3E2C6 \\ a,b,cEmail address: neuraljc@gmail.com, tn_carniello@laurentian.ca,wf_mekers@laurentian.ca
}

Keywords: heliobiology; cerebrovascular disease; space weather; solar wind; dynamic pressure; magnetic flux; solar-terrestrial interaction; cosmobiology; cycles; chronoepidemiology

\begin{abstract}
A combination of heliobiological and biometeorological perspectives on human biology and epidemiology has recently yielded many intriguing results suggesting relationships with various measures of space weather. The convergence of empirical results with quantitative dimensional analyses has also proven incredibly effective in explaining similar results and in constructing new models of solar-terrestrial interaction. In the current study, we applied these approaches to the analysis of annual mortality rates associated with cerebrovascular diseases in Canada from 1979 to 2009 to determine potential overlap with our previous work on hypertensive disease mortality with heliogeophysical factors and derive values for constructing a novel model of solar influence on terrestrial biology. Annual cerebrovascular disease mortality displayed a strong non-linear trend over time. As suspected, correlation analyses with various measures of solar, geomagnetic, and cosmic ray features suggested a significant relationship between cerebrovascular mortality and the plasma beta function for solar wind pressures which was phase shifted by $\sim 5$ to 6 years. After removing a fourth-order polynomial trend from cerebrovascular data, the dominant cycle demonstrated was $\sim 14.60$ years, offset from the $\sim 10.90$ year cycle of the average plasma beta by approximately 6 years. Finally, we employed relevant values from these results for dimensional analysis using physical constants from physics, neuroscience, and astronomy in order to help describe an Earth-Sun circuit connected by magnetic flux lines through which pressure waves might propagate, resulting in a lagged or cumulative influence on cerebrovascular-related death.
\end{abstract}

\section{INTRODUCTION}

The related fields of helio- or cosmobiology and biometeorology have now been around for a number of decades. However, prolific interdisciplinary researcher Dr. Michael A. Persinger has helped pioneer application of the most promising integrative approach to explain observed results involving a general procedure for quantification of the interactions between heliogeophysical systems and terrestrial biology. This includes the convergence of both empirical data gleaned through statistical methods and dimensional analysis of relevant physical properties. This approach has proven particularly effective for the integration of biology with the lowest orders of discourse within physics through the highest orders of astronomy and also for exploring especially novel theoretical perspectives, many of which contribute to a greater overall understanding of potential mechanisms of action for various phenomena of interest [1-9]. We previously applied a similar interdisciplinary perspective within the area of chronoepidemiology [10] which revealed a number of interesting post-hoc theoretical approaches to heliogeophysical influence on terrestrial systems. The current study was undertaken in order to help describe a novel model of solar-terrestrial interaction involving dynamic pressure systems and magnetic energies derived from empirical data and the analysis of heliogeophysical and epidemiological time-series. 
Cerebrovascular diseases account for approximately $6 \%$ of all deaths in Canada making them the third leading cause of death in the country [11]. Due to the high mortality rate associated with cerebrovascular diseases (more commonly referred to as "strokes"), a major burden is placed on the public health system and much effort has been put into early detection, treatment, and prevention [12]. As with many cardiovascular disorders, risk of cerebrovascular disease is significantly increased when an individual has hypertension or diabetes, smokes, or is obese [13]. Cerebrovascular diseases can be divided into two groups: thrombo-embolic infarcts or haemorrhagic infarcts. Thrombo-embolic infarcts occur when a blood clot formed in the heart travels to and becomes lodged in a blood vessel within the brain or when platelets gradually build up along the vessel wall and eventually occlude the blood vessel [13]. Haemorrhagic infarcts occur when a blood vessel within the brain bursts, releasing blood into the surrounding nervous tissue and increasing pressure on the brain [14]. Both forms have similar initial symptoms including headache, emesis, blurred vision, weak limbs and eventual loss of consciousness due to increased brain swelling [14]. Patients who survive often have significant neurological damage due to extended anoxic conditions. For every minute without treatment a patient can lose almost $2 \cdot 10^{6}$ neurons along with $14 \cdot 10^{9}$ synapses and one hour without treatment causes the equivalent amount of neuronal loss as approximately 3.60 years of aging [15].

The discipline of heliobiology or cosmobiology has been helpful in identifying potential heliogeophysical factors associated with terrestrial biological systems. Additional support for this area of research has been extended by the field of chronobiology in order to examine cycles associated with both biological and epidemiological measures, as well as related space weather variables. In particular, a range of relationships have been revealed between space weather parameters and human cardiovascular activity $[16,17]$ and brain activity $[18,19]$, as well as for a number of diseases including cardio- and cerebrovascular pathologies [20,21]. Of particular relevance for the current research, Zeng et al. [21] recently identified strong coperiodicities in patterns of daily mortality due to both cardio- and cerebrovascular diseases in China with a number of heliogeophysical variables including solar and geomagnetic activity, as well as the interplanetary magnetic field. Additionally, we [10] have previously described significant linear phase shifted correlations between space weather parameters, particularly related to dynamic pressure changes of solar wind, and annual hypertensive disease mortality in Canada, along with the identification of relevant overlapping cycles between measures.

Following previous approaches, we sought to examine annual deaths associated with cerebrovascular disease in Canada for linear relationships with a range of heliogeophysical parameters, as well as for related cyclic dynamics between cerebrovascular mortality and space weather. We then proceeded with values derived through empirical analyses as a potential basis to a novel quantitative model of solar-terrestrial influence incorporating universal properties and physical properties inherent to neuroscience in which the Earth-Sun system is conceived as a circuit separated by magnetic flux folds through which pressure waves might propagate.

\section{METHODS}

Procedures for epidemiological data from our previous research [10] were applied including general statistical methodology and annual disease data source. The annual total numbers of deaths associated with cerebrovascular diseases in Canada (CVD) were obtained for 1979 to 2009 for both males and females and all ages combined from the World Health Organization (WHO) online Mortality Database (http://apps.who.int/healthinfo/statistics/mortality/whodpms/) which includes ICD-10 codes I60 to I69 (Table 1). 
Table 1. International Statistical Classification of Diseases and Related Health Problems codes (version ICD-10) and corresponding medical conditions included in the World Health Organization online Mortality Database category for cerebrovascular diseases

\begin{tabular}{|cc|}
\hline ICD-10 Code & Corresponding Cerebrovascular Disease \\
\hline I60 & Non-traumatic subarachnoid hemorrhage \\
\hline I61 & Non-traumatic intracerebral hemorrhage \\
\hline I62 & Other and unspecified non-traumatic intracranial hemorrhage \\
\hline I63 & Cerebral infarction \\
\hline I65 & Occlusion and stenosis of pre-cerebral arteries, not resulting in cerebral infarction \\
\hline I66 & Occlusion and stenosis of cerebral arteries, not resulting in cerebral infarction \\
\hline I67 & Other cerebrovascular diseases \\
\hline I68 & Cerebrovascular disorders in diseases classified elsewhere \\
\hline I69 & Sequelae of cerebrovascular disease \\
\hline
\end{tabular}

Annual population estimates for Canada were obtained from the World Bank (http://data.worldbank.org/indicator/SP.POP.TOTL). Heliogeophysical variables were acquired from the Goddard Space Flight Center/Space Physics Data Facility OMNIWeb database (http://omniweb.gsfc.nasa.gov/form/dx1.html) including sunspot numbers $(S S N)$, geomagnetic activity $\left(A_{p}\right.$ index), and the solar wind plasma beta $(\beta)$ which refers to the ratio between magnetic pressure and plasma pressure. Monthly means were averaged into annual values. The average annual number of cosmic ray impulses per minute $(C R)$ were also downloaded from the Moscow Neutron Monitor (http://cr0.izmiran.rssi.ru/mosc/main.htm). The total sample analyzed consisted of $\mathrm{n}=31$ cases obtained during sunspot cycles 21, 22, and 23, including a single year (2009) from the current cycle 24 , with an average cycle length of $\sim 10.60$ years.

To additionally control for annual changes in population, a linear regression was used with CVD as the dependent variable and population as the independent variable to produce relevant residual values for further analysis. The arithmetic mean of the original (untransformed) CVD timeseries was added to each residual value in order to better reflect original units. Aside from the standard analysis of linear relationships between space weather and epidemiological measures of interest, we also employed signal processing techniques more amenable to smaller samples which included least-squares fitting of trigonometric approximations describing potential cycles in a timeseries. Where necessary, variable distributions were normalized following previous suggestion in similar contexts $[10,22]$ according to Blom's [23] method of transformation:

$$
s_{i}=\Phi\left(\left(R S_{i}-3 / 8\right) /(\mathrm{n}+1 / 4)\right)
$$

where $s=$ normalized rank score, $\Phi=$ inverse cumulative normal function, $R S=$ rank score, and $\mathrm{n}=$ sample size. However, because normalizing transformations can introduce distortions in the data, this procedure was used sparingly and only where recommended [22] for additional verification of original results related to cyclicity. Curve-fitting and non-linear analyses were performed using Matlab v7.12 while all other data transformation and statistical procedures were conducted with SPSS v17.

\section{STATISTICAL RESULTS}

Descriptive analysis of annual CVD controlling for population (mean $=14695.839$, standard deviation $=704.187$ ) and general curve-fitting procedures demonstrated a non-normal distribution with negative kurtosis ( $\mathrm{skew}=0.157$, kurtosis $=-1.526)$ and a non-linear trend over time (Figure 1 ). 


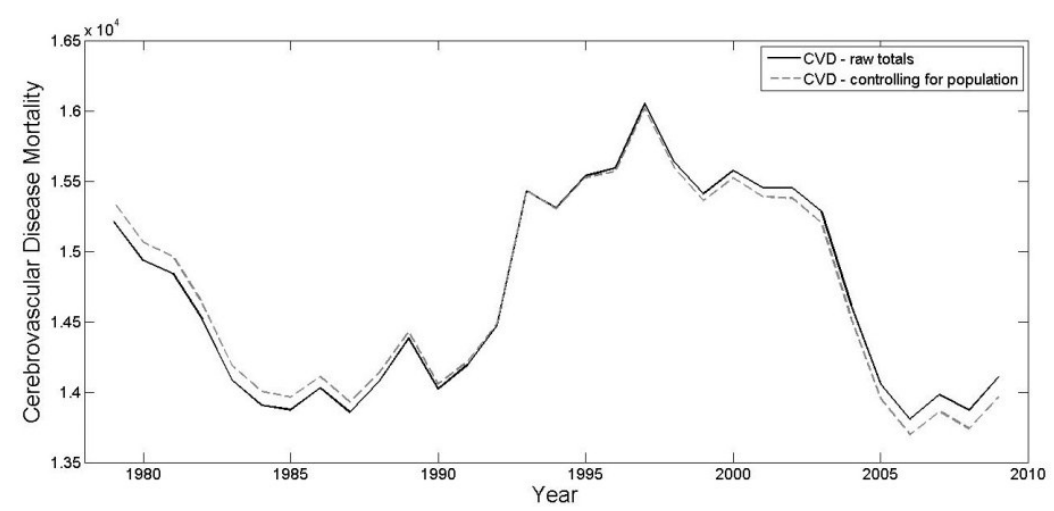

Figure 1. Chronogram of raw values for deaths related to cerebrovasculer disease (CVD) from 1979 to 2009 and residual values after controlling for annual population

All space weather measures of interest were shifted in time up to 11 years (average sunspot cycle length) in either direction $(-/+)$. All correlation analyses were tested using both parametric (Pearson $r$ ) and non-parametric (Spearman $\rho$ ) methods for further confirmation of results given the non-normal distribution of data. In addition to probability values, 95\% confidence intervals (CI) were also calculated for significant results. Symmetrical lag/lead correlations for CVD and concomitant heliogeophysical parameters revealed a singular relationship with lagged solar wind plasma beta $(\beta)$, which represents the ratio between plasma pressure and magnetic pressure, phase shifted by $\sim 5$ years $\left(\rho_{(26)}=0.480, \mathrm{CI}=0.113\right.$ to $\left.0.731, p<0.05\right)$ to 6 years $\left(\rho_{(25)}=0.483, \mathrm{CI}=0.108\right.$ to $0.737, p<0.05$ ), as demonstrated in Figure 2, which explained $\sim 20 \%$ of the variance. After examining confidence intervals and relative accuracy from curve-fitting results of the correlation(s) with low-order polynomial and power equations, it was determined that the relationship between CVD and $\beta$ lag -5 to -6 years was best fit by a power equation $\left(R^{2}=20 \%\right)$ of the form:

$$
\operatorname{CVD}(\mathrm{t})=14110 \cdot x^{0.0568}
$$

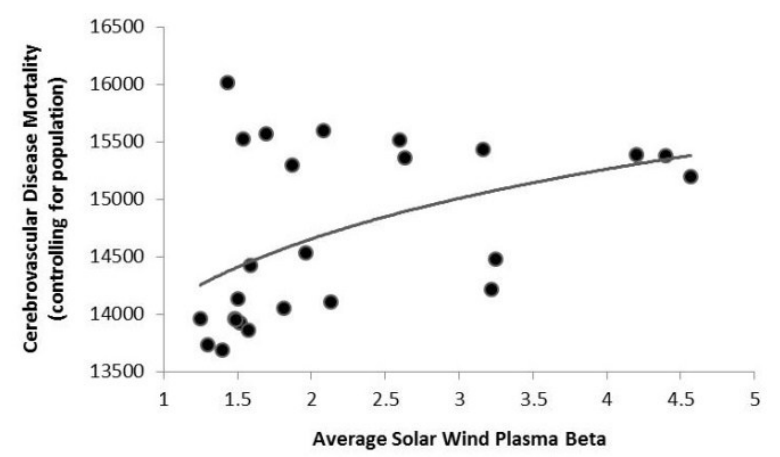

Figure 2. Scatterplot of cerebrovascular disease mortalities controlling for population (CVD) with solar wind plasma beta $(\beta)$ lagged by 6 years with line of fit from power equation

Exploratory least-squares spectral analysis using the Lomb-Scargle periodogram method was employed to investigate potential rhythmic features of CVD time series which suggested a peak periodicity of $\sim 20$ to 24 years with results confirmed using normalized values, although only a single cycle was present for the current time series employed (31 years total) and therefore this particular result was deemed too unreliable for further interpretation.

Following our previous research in this area [10], we proceeded to assess the CVD timeseries for additional periodicities after further curve-fitting and de-trending procedures common to many forms of signal processing in order to minimize the dominant cycle inherent to the data and examine potential rhythmic features buried within the overall trend. After inspection of confidence intervals (CI) and calculated root mean squared error terms (RMSE), additional curve-fitting procedures applied to CVD values controlling for population suggested a fourth-order polynomial 
trend explaining $82 \%$ of the variance (RMSE $=324$ deaths), which was removed from the data by obtaining the residuals after fitting with:

$$
\operatorname{CVD}(\mathrm{t})=0.053 \cdot x^{4}+(-4.291) \cdot x^{3}+110.60 \cdot x^{2}+(-974.50) \cdot x+16660
$$

After de-trending, data were normally distributed. Subsequent least-squares spectral analysis indicated a peak periodicity around $\sim 15$ years (Figure 3 ). Additional time structure investigation was conducted employing periodic regression with cosinor functions of the form [22]:

$$
\mathrm{CVD}(\mathrm{t})=M+\mathrm{A} \cdot \cos \left(2 \pi \mathrm{t} / T_{\mathrm{c}}\right)+\mathrm{B} \cdot \sin \left(2 \pi \mathrm{t} / T_{\mathrm{c}}\right)
$$

where $M=$ constant equivalent to a rhythm-adjusted mean, $\mathrm{A}$ and $\mathrm{B}=$ regression coefficients, $\mathrm{t}=$ time point, and $T_{\mathrm{c}}=$ cycle length in years. This suggested a more specific cyclicity of $\sim 14.60$ years $\left(R^{2}=39 \%, p=0.001\right)$ with an amplitude (approximately half of the predicted change within a period) of 269.640 annual deaths calculated according to [22]:

$$
\text { Amplitude }=\sqrt{ }\left(\mathrm{A}^{2}+\mathrm{B}^{2}\right)
$$

However, it should be noted that only two full cycles were available for the time period examined. This rhythmic feature was plotted (Figure 4) with the equation:

$$
\operatorname{CVD}(\mathrm{t})=14696.501+(-162.220) \cdot \cos (2 \pi \mathrm{t} / 14.60)+215.384 \cdot \sin (2 \pi \mathrm{t} / 14.60)
$$

As we have shown previously [10], the annual averages of $\beta$ during this period were best fit with a relatively shorter cycle length of $\sim 10.90$ years. This particular periodicity was most strongly related to the CVD cycle when offset by $\sim 6$ years.

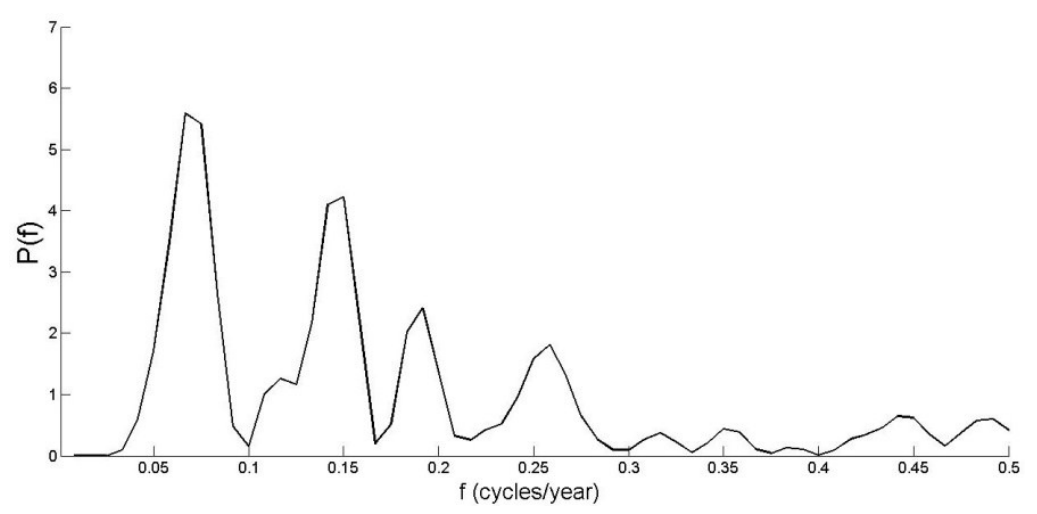

Figure 3. Periodogram of de-trended cerebrovascular disease mortality (CVD) least-squares spectral analysis with normalized power $(\mathrm{P})$ peak for a cycle length of $\sim 15$ years

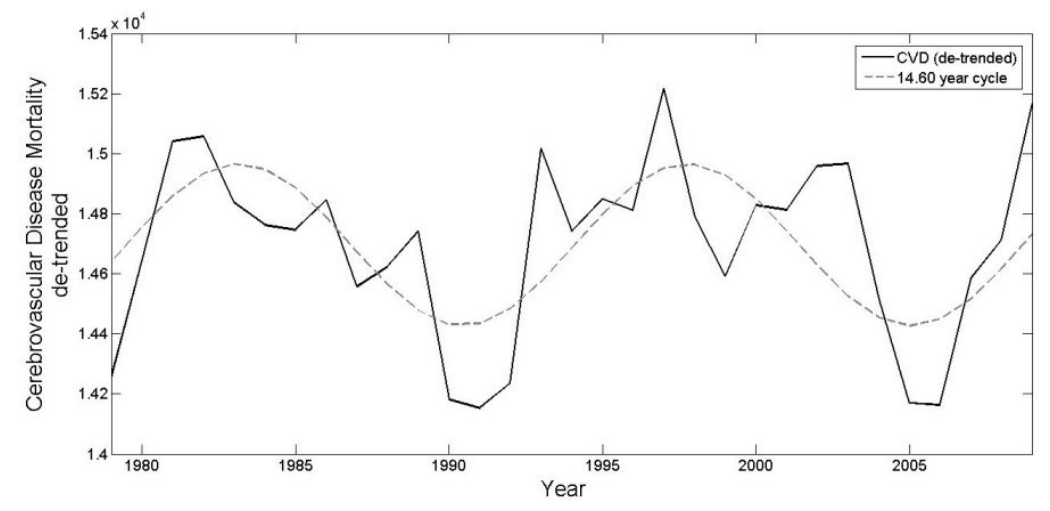

Figure 4. De-trended cerebrovascular disease mortality (CVD) time-series with superposed 14.60 year cosinor rhythm 


\section{DIMENSIONAL ANALYSIS OF A SOLAR-TERRESTRIAL CIRCUIT}

The interpretation of the cyclicity associated with cerebrovascular disease mortality as contingent upon fluctuations in $\beta$ have marked convergence upon universal constraints. For the current solar-terrestrial model of cosmoepidemiology, however, we began by isolating the temporal delay between local heliophysical fluctuations and the effect that they have on geophysical fluctuations $\sim 14.60$ years later. Magnetic reluctance within a magnetic circuit is analagous to resistance within an electrical circuit. For the current model, we postulated that the Earth and Sun are connected magnetic circuits bounded by their physical and spatial limitations. The reluctance of a magnetic circuit can be calculated using the formula:

$$
\mathrm{R}=\mathrm{L} /\left(\mu \cdot \mu_{\mathrm{r}} \cdot \mathrm{m}^{2}\right)
$$

where $\mathrm{R}=$ reluctance (turns $/ \Omega \cdot \mathrm{s}), \mathrm{L}=$ length of the circuit $(\mathrm{m}), \mu=$ permeability of free space, $\mu_{\mathrm{r}}=$ relative permeability, and $\mathrm{m}^{2}=$ surface area between circuits. We assumed that the length between the circuits is the approximate distance between the Earth and Sun $\left(\sim 1.496 \cdot 10^{11} \mathrm{~m}\right)$, the permeability of free space is $1.256 \cdot 10^{-6} \mathrm{~m} \cdot \mathrm{kg} \cdot \mathrm{A}^{-2} \cdot \mathrm{s}^{-2}$, the relative permeability for free space is 1 , and the area would be defined as a sphere with radial units equal to the distance between the Earth and Sun. The result of the calculation would be $4.24 \cdot 10^{-7}$ turns $/ \Omega \cdot \mathrm{s}$. To obtain a frequency, we multiplied this value by the wave impedance of free space $\left(3.7 \cdot 10^{2} \Omega\right)$. The resultant temporal constant for the reluctance of the Sun-Earth magnetic circuit would be $1.568 \cdot 10^{-4} \mathrm{~Hz}$. Given the known frequency associated with a single turn, we further calculated the distance that each turn (i.e., fold) in the Earth-Sun circuit would contain in order to accommodate the 14.60 year cyclicity. The equivalent duration of 14.60 years in seconds would be $4.60 \cdot 10^{8} \mathrm{~s}$ given the average year length of 365 days. The division of the cycle duration by the frequency associated with a single turn $\left(1.568 \cdot 10^{-4} \mathrm{~Hz}\right)$ would result in approximately $7.21 \cdot 10^{4}$ turns or folds in the magnetic flux separating the Earth and Sun.

The average distance occupied by a fold would be the quotient of the distance between the Earth and Sun and the number of turns. The distance a single fold occupies between the Earth and Sun is $2.07 \cdot 10^{6} \mathrm{~m}$, which is in the order of magnitude of an Earth radius, and whose coefficient differs only by a factor of approximately $\pi$, the inherent geometric conversion for a circular boundary. This may be conceptualized as wave-pockets or distortions in the magnetic field lines that connect the Earth to the Sun. Metaphorically speaking, these flux lines would be nodes upon which heliophysical perturbations vibrate and transmit patterns of energetic activity to the Earth over long periods of time. This process would require that the limit of transmission velocity be much shorter than the speed of light. However, this modulation in transmission must still obey universal constraints. The propagation velocity between the Earth and Sun along the magnetic flux folds can be calculated according to:

$$
\mathrm{v}=\mathrm{d} / \mathrm{t}
$$

where $\mathrm{v}=$ velocity, $\mathrm{d}=$ distance $(\mathrm{m})$, and $\mathrm{t}=$ time $(\mathrm{s})$. Thus, for a distance of $1.496 \cdot 10^{11} \mathrm{~m}$, and a time of $4.6 \cdot 10^{8} \mathrm{~s}$, the relative propagation velocity would be $3.25 \cdot 10^{2} \mathrm{~m} \cdot \mathrm{s}^{-1}$, or the approximate speed of sound. This may suggest that the propagation along the magnetic flux folds can be perceived as a pressure wave. From this, we may be able to find a pressure equivalent of this process.

Given that the matter density of space is approximately $10^{-27} \mathrm{~kg} \cdot \mathrm{m}^{-3}$, and that the spherical distance between the Sun and Earth is in the order of $10^{33} \mathrm{~m}^{3}$, the mass equivalence of the space between these two objects would be $10^{6} \mathrm{~kg}$. Kinetic energy can be explored as we are describing a moving wave, defined by:

$$
\mathrm{E}=1 / 2 M \mathrm{v}^{2}
$$


where $M=$ mass $\left(10^{6} \mathrm{~kg}\right)$ and $\mathrm{v}=$ velocity of the wave $\left(3.25 \cdot 10^{2} \mathrm{~m} \cdot \mathrm{s}^{-1}\right)$. The total energy involved in the propagation of a single wave over a single fold would be approximately $5.28 \cdot 10^{10} \mathrm{~kg} \cdot \mathrm{m}^{2} \cdot \mathrm{s}^{-2}$. In order to obtain a value for pressure $\left(\mathrm{kg} \cdot \mathrm{m}^{-1} \mathrm{~s}^{-2}\right)$, we simply divided by a volume. In this case, we assumed that the volume that the wave occupies extends equally in three directions and whose volume would be the cube of $2.07 \cdot 10^{6} \mathrm{~m}\left(8.87 \cdot 10^{18} \mathrm{~m}^{3}\right)$; the resultant pressure would be $5.96 \cdot 10^{-9}$ $\mathrm{kg} \cdot \mathrm{m}^{-1} \mathrm{~s}^{-2}$. This value converges upon universal background pressures calculated by dividing the approximate total energy of the universe $\left(10^{69} \mathrm{~J}\right)$ by its volume $\left(10^{78} \mathrm{~m}^{3}\right)$, as previously demonstrated by Persinger [24].

In the current study, we have presented data that suggested a 14.60 year cyclicity in CVD and a phase shifted relationship with the solar wind $\beta$ function. However, if this cycle should persist, then the epicenter of the phenomenon should be dynamic. This dynamism and associated predictable periodicity suggests a role for possible harmonic activity between the Sun and the Earth and could thus be modeled in such a manner. Given that a simple harmonic oscillator can be expressed by Hooke's law (eq. 8), we may be able to isolate a wave-force constant associated with the magnetic flux folds.

$$
\mathrm{F}=-\mathrm{kd}
$$

where $\mathrm{F}=$ force $\left(\mathrm{kg} \cdot \mathrm{m} \cdot \mathrm{s}^{-2}\right), \mathrm{k}=$ force constant $\left(\mathrm{k} \cdot \mathrm{s}^{-2}\right)$, and $\mathrm{d}=$ displacement $(\mathrm{m})$. For a force of $2.55 \cdot 10^{4} \mathrm{~N}$, derived from an energy of $5.28 \cdot 10^{10} \mathrm{~kg} \cdot \mathrm{m}^{2} \cdot \mathrm{s}^{-2}$ applied over a distance of $2.07 \cdot 10^{6} \mathrm{~m}$, and a displacement of $2.07 \cdot 10^{6} \mathrm{~m}$, the force constant would have a value of $1.237 \cdot 10^{-2} \mathrm{~kg} \cdot \mathrm{s}^{-2}$. Dividing this result by a current (A) would yield a value that represents the strength of the magnetic field in T. Given that the background energy density of the universe is $\sim 10^{-9} \mathrm{~J} \cdot \mathrm{m}^{3}$, and the volume between the Earth and Sun is $3.35 \cdot 10^{33} \mathrm{~m}^{3}$, the total background energy between the Earth and Sun would be $3.34 \cdot 10^{24} \mathrm{~J}$. Similarly, the strength of the magnetic field contained within a boundary of $3.35 \cdot 10^{33} \mathrm{~m}^{3}$ with an energy of $3.34 \cdot 10^{24} \mathrm{~J}$ can be approximated using:

$$
\mathrm{J}=B^{2} / 2 \mu V
$$

where $\mathrm{J}=$ energy in Joules, $B=$ strength of the magnetic field in $\mathrm{T}, \mu=$ permeability of free space, and $V=$ volume, and yields a value of $3.53 \cdot 10^{-8} \mathrm{~kg} \cdot \mathrm{A} \cdot \mathrm{s}^{-2}$. The quotient of the energy $\left(3.34 \cdot 10^{24} \mathrm{~J}\right)$ and the strength of the magnetic field $\left(3.53 \cdot 10^{-8} \mathrm{~kg} \cdot \mathrm{A} \cdot \mathrm{s}^{-2}\right)$ results in a magnetic moment with a value of $9.46 \cdot 10^{31} \mathrm{~A} \cdot \mathrm{m}^{-2}$. Dividing the magnetic moment by the area between the Earth and Sun $\left(2.238 \cdot 10^{22} \mathrm{~m}^{2}\right)$ results in a current with a magnitude of $4.23 \cdot 10^{9}$ A. Finally, dividing the force constant of $1.237 \cdot 10^{-2} \mathrm{~kg} \cdot \mathrm{s}^{-2}$ by the calculated current of $4.23 \cdot 10^{9}$ A results in a magnetic field strength of $2.93 \cdot 10^{-12} \mathrm{~T}$, a value which converges upon both aspects of human consciousness [25] and intergalactic magnetic field strengths [26].

To further support the convergence upon universal properties, we further calculated the change in magnetic moment under the application of an external magnetic field as defined by:

$$
\Delta m m=\left(\mathrm{e}^{2} \mathrm{r}^{2} / 4 M_{\mathrm{e}}\right) \cdot B
$$

where $\Delta m m=$ change in magnetic moment, $\mathrm{e}=$ unit charge $\left(1.6 \cdot 10^{-19} \mathrm{~A} \cdot \mathrm{s}\right), \mathrm{r}=$ Bohr radius $\left(5.11 \cdot 10^{-11} \mathrm{~m}\right), M_{\mathrm{e}}=$ mass of the electron $\left(9.11 \cdot 10^{-31} \mathrm{~kg}\right)$, and $B=$ applied magnetic field $\left(2.93 \cdot 10^{-12}\right.$ $\mathrm{T})$. The resultant change in the magnetic moment would be $5.37 \cdot 10^{-42} \mathrm{~A} \cdot \mathrm{m}^{2}$. Conceptually, the units of a magnetic moment $\left(\mathrm{A} \cdot \mathrm{m}^{2}\right)$ also reflect the relationship of $\mathrm{J} / \mathrm{T}$. Thus, one can determine an energy equivalent of an applied field as it modulates the magnetic moment of the system. For an external field with a strength of $2.93 \cdot 10^{-12} \mathrm{~T}$ and magnetic moment of $5.37 \cdot 10^{-42} \mathrm{~A} \cdot \mathrm{m}^{2}$, the energy would be equivalent to $1.57 \cdot 10^{-52} \mathrm{~J}$. Although this is seemingly an incredibly minute energy, one can consider this a rest mass (given that velocity of travel approaches unity) and can be expressed as $1.57 \cdot 10^{-52} \mathrm{~kg}$, or the approximated upper limit of the rest mass of a photon [27]. 


\section{CONCLUSIONS}

These results may suggest a potential relationship between deaths related to cerebrovascular disease (CVD) and space weather similar to the general findings from previous research [21]. However, using annual averages, the current findings could indicate that dynamic pressure changes of solar wind may better reflect any relevant heliobiological source of variation rather on a larger temporal scale compared with alternate heliogeophysical parameters often used in similar studies including overall solar or geomagnetic activity. It may also be interesting to note the previously observed overlapping relationships for both cardio- and cerebrovascular mortality with space weather using daily averages in China [21], along with our recent findings for annual hypertensive deaths in Canada [10], the latter of which also strongly implicated the solar wind plasma beta $(\beta)$ as the most likely source of variance regarding these relationships.

Although we may not infer causation from correlation, the accumulation of empirical evidence that has converged upon similar conclusions as those observed here nonetheless provides an interesting avenue for further discussion and quantification. Furthermore, while the coefficients obtained were relatively weak ( $20 \%$ variance explained), the current range is generally reflective of that observed in many additional heliobiological studies [28-29] and the dominant relationship with $\beta$ among all space weather variables employed is also in line with our previous examination of annual hypertensive disease mortality [10]. Even a seemingly miniscule amount of variance explained can be quite significant in the context of a large population. Vares and Persinger [29] have aptly noted that biometeorological effects on mood and sociological phenomena typically explain only $\sim 10 \%$ of the variance. However, small effects applied to large populations of individuals can accumulate to produce notable changes. It remains important to clarify that these results, overall, may not appear as strong as those observed in some larger studies from this area. However, it should also be stated that a majority of the available helioepidemiological research has been conducted in Eastern European and Middle Eastern regions compared to other areas, and has also typically employed different temporal resolutions for the data examined (e.g., daily). Despite these discrepancies, the current results obtained using annual data with a North American population have presented a number of intriguing relationships congruent with other related studies, and from which the solar-terrestrial circuit model of heliobiological influence has been partially derived.

As previously discussed, solar wind $\beta$ was identified as the most likely source of heliogeophysical influence, congruent with our recent findings for mortality due to hypertensive disease [10]. The overall non-linear pattern in CVD was significantly related to lagged solar wind pressure dynamics phase shifted by $\sim 5$ to 6 years, while the dominant cycle identified for annual CVD after curve-fitting and de-trending procedures was $\sim 14.60$ years. Although our subsequent calculations contributing to the model are largely exploratory, the possible relationships described here may aid further research into the potential effects of space weather on terrestrial biology and epidemiology through an inherently interdisciplinary perspective employing concepts from physics and astronomy.

\section{Acknowledgments:}

The authors would like to thank Prof. Nicolas Rouleau for internal review and editing. We also wish to thank Dr. Karel Kudela for ongoing illuminating discussions and Dr. Natalia Papitashvili for maintaining the SPDF OMNIWeb database. Additional thanks are due to two anonymous reviewers from a previous draft of this manuscript for further suggestions. This work is dedicated to Dr. Michael A. Persinger, brilliant interdisciplinary scientist and mentor. 


\section{References}

[1] L. M. Karbowski, M. A. Persinger, International Letters of Chemistry, Physics and Astronomy 43 (2015) 1-9.

[2] M. A. Persinger, Open Biology Journal 6 (2013) 8-13.

[3] M. A. Persinger, International Letters of Chemistry, Physics and Astronomy 8 (2013) 8-19.

[4] M. A. Persinger, International Letters of Chemistry, Physics and Astronomy 2 (2014) 15-21.

[5] M. A. Persinger, International Letters of Chemistry, Physics and Astronomy 11 (2014) 24-32.

[6] M. A. Persinger, International Letters of Chemistry, Physics and Astronomy 20 (2014) 160-165.

[7] M. A. Persinger, R. M. Lafrenie, International Letters of Chemistry, Physics and Astronomy 17 (2014) 67-77.

[8] M. A. Persinger, K. S. Saroka, Journal of Signal and Information Processing 6 (2015) 153-164.

[9] K. S. Saroka, M. A. Persinger, International Letters of Chemistry, Physics and Astronomy 20 (2014) 166-194.

[10] J. M. Caswell, T. N. Carniello, N. J. Murugan, International Journal of Biometeorology (2015).

[11] Statistics Canada, The 10 Leading Causes of Death, 2011, Retrieved from http://www.statcan.gc.ca/pub/82-625-x/2014001/article/11896-eng.htm (2014).

[12] T. Olalla, J. A. Medrano, J. Sierra, J. Almazán, European Journal of Epidemiology 15 (1999) 331-339.

[13] T. J. Fowler, R. W. May, Neurology (1985) Netherlands: Springer Netherlands.

[14] S. S. Rathore, A. R. Hinn, L. S. Cooper, H. A. Tyroler, W. D. Rosamond, Stroke 33 (2002) 2718-2721.

[15] J. L. Saver, Stroke 37 (2008) 263-266.

[16] S. Dimitrova, F. R. Mustafa, I. Stoilova, E. S. Babayev, E. A. Kazimov, Advances in Space Research 43 (2009) 641-648.

[17] M. Papailiou, H. Mavromichalaki, K. Kudela, J. Stetiarova, S. Dimitrova, Natural Hazards 61 (2012) 719-727.

[18] B. P. Mulligan, M. A. Persinger, Neuroscience Letters 516 (2012) 54-56.

[19] K. S. Saroka, J. M. Caswell, A. Lapointe, M. A. Persinger, Neuroscience Letters 560 (2014) 126-130.

[20] G. Cornélissen, F. Halberg, T. Breus, E. V. Syutkina, R. Baevsky, A. Weydahl, Y. Watanabe, K. Otsuka, J. Siegelova, B. Fiser, E. E. Bakken, Journal of Atmospheric and Solar Terrestrial Physics 64 (2002) 707-720.

[21] W. Zeng, X. Liang, C. Wan, Y. Wang, Z. Jiang, S. Cheng, G. Cornélissen, F. Halberg, Z. Wang, Biological Rhythm Research 45 (2014).

[22] G. Cornélissen, Theoretical Biology and Medical Modelling 11 (2014) 16-40.

[23] G. Blom, Statistical Estimates and Transformed Beta Variables (1958) New York, New York.

[24] M. A. Persinger, International Journal of Astronomy and Astrophysics 4 (2014). 
[25] M. A. Persinger, Journal of Cosmology 14 (2011).

[26] A. Neronov, I. Vovk, Science 328 (2009) 73-75.

[27] L. C. Tu, J. Luo, G. T. Gilles, Reports on Progress in Physics 68 (2005) 77.

[28] M. Kancírová, K. Kudela, Journal of Astrobiology and Outreach 2 (2014).

[29] D. A. E. Vares, M. A. Persinger, Global Journal of Human Social Science: B 15 (2015). 